\title{
Retinoschisin, a Photoreceptor-Secreted Protein, and Its Interaction with Bipolar and Müller Cells
}

\author{
Silvia N. M. Reid, ${ }^{1}$ Clyde Yamashita, ${ }^{1}$ and Debora B. Farber ${ }^{1,2}$ \\ ${ }^{1}$ Jules Stein Eye Institute, University of California Los Angeles School of Medicine, Los Angeles, California 90095, and 2Molecular Biology Institute, \\ University of California Los Angeles, Los Angeles, California 90095
}

\begin{abstract}
Usually, photoreceptors interact with other retinal cells through the neurotransmitter glutamate. Here we describe a nonsynaptic interaction via a secreted protein, retinoschisin. Using in situ hybridization, we found that from early postnatal life retinoschisin mRNA is present only in the outer retina of the mouse, and with single-cell RT-PCR we demonstrated its localization in rod and cone photoreceptor cells but not in Müller cells. Western blot analyses of proteins from cultured ocular tissues and microdissected outer and inner retinas, as well as from the culture media of these samples, showed that retinoschisin is secreted from the photoreceptor cells. Immunostaining of permeabilized and nonpermeabilized dissociated retinal cells revealed that retinoschisin is mainly inside and outside the photoreceptors, outside bipolar cells, and associated with plasma membranes of Müller cells and inside their distal processes. Because we showed previously that retinoschisin is distributed all over the retina, our current data suggest that after synthesis and secretion by the photoreceptors, retinoschisin reaches the surface of retinal cells and mediates interactions/adhesion between photoreceptor, bipolar, and Müller cells, contributing to the maintenance of the cytoarchitectural integrity of the retina. These interactions may not occur when the gene encoding retinoschisin is mutated, as it occurs in X-linked juvenile retinoschisis, a disease that results in morphological and electrophysiological defects of the retina.
\end{abstract}

Key words: photoreceptor; glia; neurodegenerative disease; retinal disease; retinal development; neurodegeneration; bipolar cell; protein secretion; cell interaction; retinoschisis; Müller cell

\section{Introduction}

Signaling molecules are intricately woven into the nervous system communication network. In addition to neurotransmitters, extracellular signaling molecules such as adhesion proteins and trophic factors also participate in cell-cell interactions for facilitation of synaptic transmission, neurite growth, axon guidance, cell migration, and cell differentiation. Neuropilin-1, neurexin IV, contactin-associated protein, SCO-spondin, and tyrosine protein kinase Ptk-3-proteins involved in axon-pathfinding, cell migration, cell aggregation, and cell interactions with gliaall contain the FA58C/discoidin domain (Sánchez et al., 1994; Baumgartner et al., 1996; Gobron et al., 1996; Einheber et al., 1997; He and Tessier-Lavigne, 1997; Kitsukawa et al., 1997; Kolodkin et al., 1997; Peles et al., 1997; Marín et al., 2001) that has been described in the InterPro database to function in cell-cell adhesion (Apweiler et al., 2001). Because the FA58C/discoidin domain makes up $\sim 70 \%$ of the retinal protein retinoschisin

\footnotetext{
Received Dec. 30, 2002; revised April 8, 2003; accepted April 18, 2003.

This work was supported by National Institutes of Health Grant EY08285. D.B.F. is a recipient of a Research to Prevent Blindness Senior Investigators Research Award. We thank Drs. Dorothy Trump, John Saari, Shinobu Fujita, and Dean Bok for antibodies, Dr. Gabriel Travis for his helpful discussion, and Dr. Craig Reid for assistance with this manuscript. We also thank Dr. Alapakkam Sampath for isolation of retinal cells for RT-PCR, Dr. Matthew Schibler for his assistance in confocal microscopy, and Tawny Saleh for her assistance in the immunostaining of dissociated cells. Correspondence should be addressed to Silvia N. M. Reid, Jules Stein Eye Institute, University of California Los Angeles School of Medicine Center for the Health Sciences, 100 Stein Plaza, Los Angeles, CA 90095-7008. E-mail: reiddrs@aol.com.

Copyright $\odot 2003$ Society for Neuroscience $\quad$ 0270-6474/03/236030-11\$15.00/0
}

(Sauer et al., 1997; Reid et al., 1999), studies on this protein will provide insights into cell-cell interactions in the retina.

Mutations in the gene encoding retinoschisin cause X-linked juvenile retinoschisis (XLRS) (Sauer et al., 1997; The Retinoschisis Consortium, 1998). This disease is characterized by pinwheelshaped folds in the fovea and a reduced b-wave in the electroretinogram (ERG) (George et al., 1995) that reflects defective activity in the inner retina (Peachey et al., 1987). The XLRS retina displays macular atrophy, microcysts present mainly in the macular region, separation of the nerve fiber layer/inner limiting membrane from the remaining retina, and accumulation of extracellular filaments in retina and vitreous (Yanoff et al., 1968; Manschot, 1972; Condon et al., 1986). It has been speculated that Müller cells, the principal glial cells of the vertebrate retina, are responsible for XLRS (Condon et al., 1986). However, recent evidence attributed the physiological defects of XLRS to bipolar cells (Alexander et al., 2000; Shinoda et al., 2001).

Retinoschisin is found throughout the retina (Grayson et al., 2000; Molday et al., 2001), whereas the mRNA required for its synthesis is present only in the outer retina, mainly in the photoreceptor inner segments (Reid et al., 1999). The association of retinoschisin with photoreceptors was first reported by Grayson et al. (2000) and was confirmed by Molday et al. (2001). Because retinoschisin contains a secretory leader peptide (Sauer et al., 1997; Reid et al., 1999) and is in the culture media of photoreceptor-like retinoblastoma cells (Grayson et al., 2000), we hypothesized previously that after its secretion from photore- 
ceptors, retinoschisin interacts with inner retinal cells to maintain the retinal cytoarchitecture (Reid et al., 1999; Grayson et al., 2000). However, immunostaining results led Molday et al. (2001) to propose that retinoschisin is also synthesized by the inner retinal bipolar cells. In this paper, we document the disparate distribution of retinoschisin mRNA (confined to the photoreceptors) and protein throughout postnatal development of the mouse retina. We also demonstrate retinoschisin secretion from photoreceptor cells and its localization on the surface of bipolar and Müller cells. These data suggest that photoreceptor-secreted retinoschisin mediates nonsynaptic interactions between retinal cells.

\section{Materials and Methods}

\section{Tissue preparation}

The eyes of normal C57BL/6J and $r d / r d$ mice were prepared for histology either after perfusion as described previously (Reid et al., 1999) or immersion-fixation in buffered $4 \%$ paraformaldehyde. Eyes without fixation were used for cell dissociation and nonhistological procedures. The use of animals in the experiments was in accordance with the guidelines approved by The Society for Neuroscience.

\section{Cell dissociation}

The method described by Ishii et al. (1997) was used with minor modifications to enzymatically dissociate retinal cells. Eyecups were digested $\left(37^{\circ} \mathrm{C}, 10\right.$ or $\left.20 \mathrm{~min}\right)$ in papain $(1 \mathrm{mg} / \mathrm{ml})$. After removal of the retinal pigment epithelium (RPE)/choroid/sclera (R/C/S), retinas were further digested $\left(37^{\circ} \mathrm{C}, 20 \mathrm{~min}\right)$. Dissociated cells were collected by centrifugation at $80 \times g$ and fixed in buffered $4 \%$ paraformaldehyde.

\section{In situ hybridization}

The detection of mouse retinoschisin gene (Xlrs1) expression by using digoxigenin (DIG)-tagged sense- and antisense-cRNA probes (bp 1698 4488 of Xlrs1) was performed as described previously (Reid et al., 1999) on cryosections of eyecups ( 10 or $16 \mu \mathrm{m}$ thick). The final alkaline phosphatase reaction was performed for $2 \mathrm{hr}$ or for $10.5 \mathrm{hr}$ to enhance detection of low levels of mRNA.

\section{Immunostaining of retinal sections and cells}

Antibodies. RS24-37 (a generous gift from Dr. D. Trump, Cambridge Institute for Medical Research, Cambridge, UK), an affinity-purified rabbit polyclonal antibody, was raised against a synthetic peptide corresponding to amino acid residues 24-37 of retinoschisin (aa 24-37) (Grayson et al., 2000). UW55 (kindly provided by Dr. John Saari, University of Washington, Seattle, WA), a rabbit polyclonal antibody, was raised against a Müller cell protein, cellular retinaldehyde-binding protein (CRALBP) (Eisenfeld et al., 1985). ROB (115A10; a kind gift of Dr. Shinobu C. Fujita, Mitsubishi Kasei Institute of Life Sciences, Machida, Japan), a mouse monoclonal antibody, recognizes a cell surface protein of bipolar cells (Onoda and Fujita, 1987; Haverkamp and Wässle, 2000). All secondary antibodies were purchased from Jackson ImmunoResearch (West Grove, PA), if not noted otherwise.

Immunocytochemistry. The immunodetection of retinoschisin using RS24-37 (1:250) and a biotin-avidin-peroxidase-based method was performed as described previously (Grayson et al., 2000). RS24-37 preabsorbed with synthetic peptide aa $24-37(0.2 \mu \mathrm{g} / \mu \mathrm{l})$ for $3 \mathrm{hr}\left(25^{\circ} \mathrm{C}\right)$ was used for control staining.

Indirect immunofluorescence. Methanol-permeabilized $\left(-80^{\circ} \mathrm{C}, 3\right.$ $\mathrm{min})$ and nonpermeabilized retinal cells immunoreacted with RS24-37 (1:250) and UW55 (1:1000) in $1 \%$ normal rabbit serum at $4^{\circ} \mathrm{C}$, overnight, were visualized after a $2 \mathrm{hr}$ incubation at $25^{\circ} \mathrm{C}$ with indocarbocyanine (Cy3)-conjugated donkey anti-rabbit $\operatorname{IgG}(1: 200)$ in $1 \%$ normal donkey serum. RS24-37 preabsorbed with aa 24-37 $(0.1 \mu \mathrm{g} / \mu \mathrm{l})$ was used as a control. For ROB staining, proteinase K-permeabilized or nonpermeabilized cells were incubated in ROB (1:100 in normal donkey serum/bovine serum albumin, $4^{\circ} \mathrm{C}$, overnight) and visualized with a fluorescein isothiocyanate (FITC)-conjugated donkey anti-mouse IgG (1:200).

In sequential immunostaining experiments (to avoid the influence of bleed-through on simultaneous double labeling), retinoschisin-immunopositive cells were further stained for other proteins. Permeabilized Müller cells were stained with ROB and later with UW55 $\left(25^{\circ} \mathrm{C}, 3.5 \mathrm{hr}\right)$ without additional permeabilization. Nonpermeabilized, retinoschisin-positive cells were incubated with either ROB or UW55 (1:500). The UW55-treated cells were then permeabilized with $0.5 \%$ Triton X-100, stained with ROB, and incubated again with UW55 (1:500). Each staining was performed only after immunofluorescence images of the previous staining had been acquired. FITC-conjugated donkey IgG was used to visualize the UW55-positive and ROB-positive cells.

The pattern of retinoschisin immunofluorescence was compared with those of FM 1-43 (Molecular Probes, Eugene, OR)-labeled cell membranes (Whalley et al., 1995) and 7-amino-4-chloromethylcoumarin (CellTrack Blue CMAC, Molecular Probes)-stained cytosols (MuñozBarroso et al., 1998). Enzymatically dissociated cells were washed with DMEM, incubated with $8 \mu \mathrm{M} \mathrm{CMAC}$ for $15 \mathrm{~min}$ at $37^{\circ} \mathrm{C}$, and then incubated with $3 \mu \mathrm{M}$ FM $1-43$ for $15 \mathrm{~min}$ at $37^{\circ} \mathrm{C}$. After fixation in $4 \%$ paraformaldehyde, the labeled cells were smeared onto glass slides, immunoreacted with RS24-37 $\left(1: 1000,4^{\circ} \mathrm{C}\right.$, overnight $)$, and labeled with Alexa Fluor 488-conjugated goat anti-rabbit IgG (Molecular Probes). The cell images were acquired with the aid of a Leica DM IRBE microscope equipped with a Leica TCS-SP confocal component.

Quantification of immunofluorescence. Retinoschisin immunofluorescence images were acquired from randomly picked photoreceptor, bipolar, and Müller cells enzymatically dissociated from retinas of two mice. One single confocal optical section was recorded across the center of each cell, under identical settings, using a Zeiss LSM 10 confocal microscope. The structure of interest was selected by outlining it with the Lasso tool, and, without any image modification, the averaged fluorescence intensity per pixel (FI) of the outlined structure was measured on a scale of $0-255$ using the luminosity channel of the histogram function in Adobe Photo Shop. The background fluorescence intensity (BI) was acquired from an adjacent blank area. The FI of each structure was converted into the relative fluorescence intensity $(\mathrm{RI})$, defined as: $\mathrm{RI}=(\mathrm{FI}-\mathrm{BI}) \times 100 / \mathrm{BI}$. The average of all the RIs for each structure of the cell stained with RS24-37 preabsorbed with aa 24-37 was used as the standard (S) for that structure. The relative level of retinoschisin immunofluorescence (RL) for each structure of each cell was obtained as: $R L=(R I-S) \times 100 / S$. The RLs were analyzed separately for each cell type by three-way factorial ANOVA, using antibody treatment, permeabilization procedure, and structures of the cell as the three analytical factors. Tukey HSD multiple comparison was used for the post hoc analyses.

\section{Single-cell RT-PCR}

Retinal cells were mechanically dissociated by lightly chopping flattened retinas in PBS with a razor blade according to Sampath et al. (1998). Individual retinal cells were drawn into separate micropipettes with the aid of an inverted microscope. The isolated cells were then subjected to RT-PCR as described by Kumazaki et al. (1994) using $r$ Tth DNA polymerase (PerkinElmer Life Sciences, Branchburg, NJ) with some modifications. Each RT reaction product was divided for further PCR detection of specific cDNAs in the presence of appropriate primers. Primers GGTACCAGAAAGCATGCAA (exon 3) and GCTCCATCCGGATGGCAATT (exon 6) were used for Xlrs1 mRNA; primers CTGAGAAGGATGCCCGCACTG (exon 1) and CTCACCTGCCGGGCTGAATCT (exon 3) were used for the rod-specific $\alpha_{1}$-subunit of transducin $(R T \alpha)$; primers TAGAGTTCAAGTCTGTCATCT (forward) and CTGCTCGTTAGGGAGGTAGTT (reverse) were used for the cone-specific $\alpha_{2}$ subunit of transducin $(C T \alpha)$; and primers CCGCCTCTGCTGGAATGTGT (exon 6) and CGGCTGAGCTGGACGCCAGTT (exon 7) were used for carbonic anhydrase II (CAII), yielding fragments of 549, 269, 317 , and 150 bp that identified Xlrs1 mRNA, rod, cone, and Müller cells, respectively. These first-round PCR products were subjected to Southern blot analyses or another round of PCR. Gel-purified, ${ }^{32} \mathrm{P}$-labeled probes generated by RT-PCR of total mouse retinal RNAs using the above primers and the multiprimer DNA labeling system (Amersham Biosciences, Piscataway, NJ) were used in Southern blot analyses. 


\section{Secretion experiments}

Three experiments were performed. In the first, enucleated eyes of 12 adult mice were used to obtain anterior segment, eyecup, $\mathrm{R} / \mathrm{C} / \mathrm{S}$, and retina. For each tissue type, 12 individual samples were pooled and divided into two sets. Each duplicate was placed in a separate well of a tissue-culture plate, washed, and incubated with $300 \mu \mathrm{l}$ of DMEM in a humid-chamber equilibrated with $95 \% \mathrm{O}_{2} / 5 \% \mathrm{CO}_{2}$.

In the second experiment, individual retinas of 73- and 139-d-old $r d / r d$ mice and two normal mice were incubated singly in $200 \mu \mathrm{l}$ of DMEM under the conditions stated above.

In the third experiment, we examined retinoschisin secretion from the inner and outer retinas. Retinas of normal adult mice were flattened on a polytetrafluoroethylene Biopore membrane (Millipore, Bedford, MA), placed on a gelatin stage mounted on a vibratome, and embedded onto the stage with $4 \%$ gelatin. A $100-\mu \mathrm{m}$-thick retinal section was cut; the $150-\mu \mathrm{m}$-thick section containing the rest of the retina was then removed from the stage. Both sections were washed and incubated separately in $200 \mu \mathrm{l}$ of DMEM under the same conditions as before.

At the end of $\sim 22.5,27$, and $37 \mathrm{hr}$ incubation at $25^{\circ} \mathrm{C}$ for the first, second, and third set of experiments, respectively, the medium and tissue from each well were collected for Western blot analyses.

\section{Western blot analyses}

Sample preparation. Tissues were homogenized as described previously (Reid et al., 1997). After centrifugation at $1000 \times \mathrm{g}\left(10 \mathrm{~min}, 4^{\circ} \mathrm{C}\right)$, the supernatant was used in Western blots or processed further for cellular fractionation. The cytosolic fraction was obtained by collecting the supernatant after $1 \mathrm{hr}$ centrifugation at $100,000 \times g\left(4^{\circ} \mathrm{C}\right)$. The pelleted membrane was lysed in $10 \mathrm{~mm}$ Tris, $\mathrm{pH} 7.4,1 \mathrm{~mm}$ EDTA, with the protease inhibitors initially used, homogenized, and further pelleted at $100,000 \times g\left(4^{\circ} \mathrm{C}\right)$.

Immunoblotting. Protein $(10 \mu \mathrm{g})$ from tissue homogenate [protein concentration determined by the method of Peterson (1977)] was denatured in 2\% SDS in Tris, pH 6.8, 0.01\% bromophenol blue, 10\% glycerol, with or without $50 \mathrm{~mm}$ dithiothreitol (DTT), and then separated by SDS-PAGE on 4-20\% gradient gels. Culture media collected from each well of the tissue culture plate from the secretion experiments described above was filtered (Millipore Ultrafree-MC filter with 0.1 or $0.2 \mu \mathrm{m}$ pore size). Twenty microliters from each well were incubated with SDS- and DTT-containing buffer before SDS-PAGE.

Proteins in gels were electroblotted onto polyvinylidene difluoride membranes. The membranes were incubated with one of the following antibodies: RS24-37 (1:500), anti-rod rhodopsin (1:1000), and antiinterphotoreceptor retinoid-binding protein (IRBP) (1:10,000; a gift from Dr. D. Bok, University of California Los Angeles) and processed for an alkaline phosphatase-based enhanced chemiluminescence detection (DuoLux) following the protocol recommended by the vendor (Vector Laboratories, Burlingame, CA).

\section{Results \\ Distribution of retinoschisin mRNA and protein during postnatal development of normal mouse retina}

The mismatched distribution of retinoschisin mRNA and protein in the adult mouse retina that we described previously (Reid et al., 1999; Grayson et al., 2000) may not occur during development. Therefore, retinoschisin in the adult inner retina could be the remainder of protein produced earlier. To test this hypothesis and determine the time course of appearance and location of retinoschisin mRNA in mouse retina, experiments were performed using in situ hybridization. Retinoschisin mRNA signals were detected in retinal sections reacted with the antisense probe at all ages except postnatal day (P) 3, whereas no signal was found in any section reacted with the sense probe (Fig. $1 \mathrm{~A}$ ). Retinoschisin mRNA was not detected at P3 even after a prolonged reaction ( $10.5 \mathrm{hr}$ ), but a weak signal in the outer neuroblastic zone at the scleral margin was revealed in the P5 retina. Stronger mRNA expression with a sclera-to-vitreous gradient of intensity across the outer nuclear layer (ONL) that stopped before the outer plexiform layer (OPL) was observed from P6 to P14, with a dramatic increase in intensity between P6 and P7 (Fig. 1A). The signal in the inner half of the ONL became more apparent at P14, especially after a prolonged reaction, and was most prominent at P21 (Fig. 1A). In the adult retina (P82), retinoschisin mRNA was present in the entire ONL, but it was concentrated mainly in the inner segment of the photoreceptors. No mRNA signal was detected in the inner retina at any age even after a $10.5 \mathrm{hr}$ reaction. The time course of retinoschisin mRNA appearance and its distribution are consistent with our reported Northern blot results (Reid et al., 1999).

Retinoschisin immunoreactivity was found at all examined ages when retinal sections were treated with the RS24-37 antibody except for P3, but it was not observed when the antibody was preabsorbed with the aa 24-37 peptide against which it had been raised (Fig. $1 A$ ). The brown immunoreaction product was noticeable at P5 in the sclerad third of the neuroblastic zone with the highest intensity at the scleral margin, and it spread farther inward at P6. By P7, retinoschisin had occupied the entire ONL, the inner plexiform layer (IPL) and the nerve fiber layer (NFL), and at P8 it became very clear in the inner nuclear layer (INL). At P14, after eye opening, retinoschisin was seen all over the retina but was concentrated mostly in the area of the photoreceptor inner segments (Fig. 1A). A similar pattern was also found in the P21 and adult retinas, with staining of the OPL and the INL increasing with age. These results are consistent with our previous findings in adult mouse and human retinas (Grayson et al., 2000); furthermore, detection of retinoschisin in the plexiform layers has also been confirmed by Molday et al. (2001) and Weber et al. (2002).

In the adult mouse, the retinoschisin-immunoreactive product outlined the inner segment of the photoreceptors and appeared most intense immediately adjacent to the outer segment (Fig. 2a). The product formed patches around cell bodies in the INL, decorated the processes in the IPL (Fig. 2b), and was associated with structures that emulated the end feet of Müller cells in the NFL (Fig. 2c).

\section{Distribution of mRNA and protein in the degenerating retinas of $r d / r d$ mice}

$r d / r d$ mice are affected with an inherited retinal degeneration characterized by the rapid loss of photoreceptor cells during early postnatal life. Therefore, we did not observe a photoreceptor layer in retinas of P73 and P139 rd/rd mice. Consistently, we did not detect retinoschisin mRNA by in situ hybridization in these retinas even after $10.5 \mathrm{hr}$ of alkaline phosphatase reaction (Fig. $1 B)$. This prolonged reaction did reveal, however, retinoschisin mRNA in P5 and P6 normal retinas (Fig. 1A) that have a transcript level barely detectable on Northern blots (Reid et al., 1999). Faint immunostaining of retinoschisin could still be found in patches of the remaining retina of the 73-d-old $r d / r d$ mouse (Fig. $1 B$ ). These patches became less frequent and very faint but were still seen in the $r d / r d$ INL at P139, consistent with the results of Molday et al. (2001).

\section{RT-PCR detection of Xlrs1 expression}

Because the distal processes of Müller cells extend across the ONL, the Xlrs 1 mRNA seen by in situ hybridization in this layer could be present not only in photoreceptors but also in Müller cell processes. To test this possibility, we amplified retinoschisin mRNA from individual retinal cells using RT-PCR (Fig. 3). The identity of each cell was unequivocally determined by the expres- 


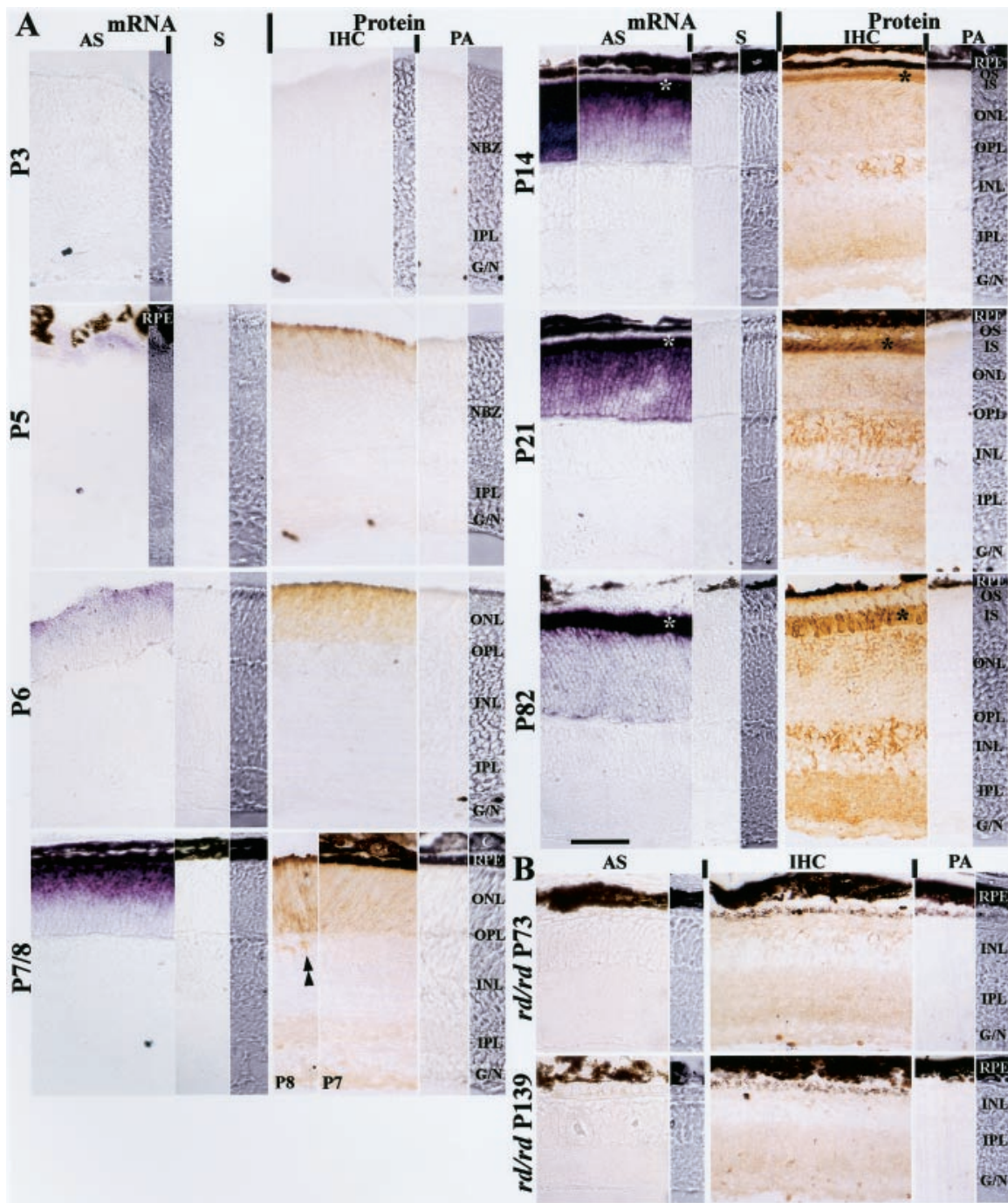

Figure 1. Distribution of retinoschisin $m$ RNA and protein in normal $(A)$ and $r d / r d$ mouse $(B)$ retinas. $A$, Retinoschisin mRNA was detected with a digoxigenin-labeled antisense-CRNA probe (AS) and visualized by the purple reaction product of the alkaline phosphatase reaction; no staining was observed with sense-cRNA probe (S) used as control. Retinoschisin was detected by immunohistochemistry (IHC); the brown peroxidase-reaction product was observed after immunoreaction with an affinitypurified polyclonal antibody against amino acid residues $24-37$ of retinoschisin (aa $24-37$ ). This antibody was preabsorbed with aa 24-37 and used as control (PA). NBZ, Neuroblastic zone; IPL, inner plexiform layer; $G / N$, ganglion cell and nerve fiber layers; RPE, retinal pigment epithelium; ONL, outer nuclear layer; OPL, outer plexiform layer; INL, inner nuclear layer; $C$, choroid; OS, outer segment of photoreceptors; IS, inner segment of photoreceptors. Retinoschisin mRNA and protein were not detected in the retina of normal mice at P3 but were found at a very low level at P5. A mismatched mRNA and protein localization started on P7 and persisted into adulthood (P82). Retinoschisin mRNA was in the outer retina throughout postnatal development (outer NBZ on P5; ONL on P6 and P7/8; IS and ONL on P14, 21, and 82). In contrast, retinoschisin (Protein) was initially in the outer retina (outer NBZ on $\mathrm{P} 5$ and $0 \mathrm{NL}$ on P6) and then appeared in the inner retina. Immunopositive cell bodies in the INL (double arrowheads) started appearing at P8 and showed increased intensity as the retina matured. By P21, retinoschisin was abundant in all layers. Both $\mathrm{mRNA}$ and protein were most concentrated in the area corresponding to the photoreceptor inner segments (*). Visualization of the mRNA was achieved with a $2 \mathrm{hr}$ alkaline phosphatase reaction $(P 7 / 8,14$, and 21$)$ or $10.5 \mathrm{hr}(\mathrm{P3}, 5,6$, and 82 , and the left micrograph in the AS column for P14). B, At P73 and P139, most photoreceptors had degenerated in the $r d / r d$ retinas, and retinoschisin mRNA was not detected even after $10.5 \mathrm{hr}$ of reaction. Faint retinoschisin staining was present in the INL, the IPL, and the $\mathrm{G} / \mathrm{N}$. The micrograph strips at the far right of the mRNA and protein panels for each age are high-contrast bright-field micrographs taken from the same section as the adjacent micrographs. High-contrast micrographs were also taken from a portion of the sections in the AS panel at P3, the IHC panel at P3, and the AS panel at P5. Scale bar: for all micrographs, $50 \mu \mathrm{m}$.

subjecting these cells to RT-PCR, the expression of all four genes was observed (Fig. 3). Cells that had no structural resemblance to photoreceptor and Müller cells were isolated as negative controls (Fig. 3, NC); no expression was found in them for any of the four genes (Fig. 3).

\section{Retinoschisin in the retina and cultured ocular tissues}

Similar to our previous results (Grayson et al., 2000), two protein bands from retinal homogenates treated with the disulfidereducing agent DTT were recognized on Western blots by the antibody RS24-37 against retinoschisin. These proteins had apparent molecular masses of $27 \mathrm{kDa}$ (slightly higher than the mass predicted from the deduced amino acid sequence of retinoschisin) and $65 \mathrm{kDa}$ (Fig. $4 \mathrm{~A}$, first lane of $\mathrm{H}$ in the left panel). In the absence of DTT, the $65 \mathrm{kDa}$ band and smears of higher molecular mass were detected, but not the $27 \mathrm{kDa}$ band (Fig. $4 A$, second lane of $\mathrm{H}$ in the left panel). After cellular fractionation, the $65 \mathrm{kDa}$ protein was found in the nonreduced cytosol fraction (C) but not in the nonreduced membrane preparation $(\mathrm{M})$, whereas the smear was present predominantly in the nonreduced membrane fraction (Fig. 4A). When the membrane preparation was incubated at room temperature for $1 \mathrm{hr}$ with DTT, only the 27 $\mathrm{kDa}$ protein was labeled by the RS24-37 antibody; in contrast, treatment of the cytosol with DTT did not change the $65 \mathrm{kDa}$ protein (Fig. $4 B$, left panel). The antibody no longer labeled the 65 and $27 \mathrm{kDa}$ proteins after it had been preabsorbed with the synthetic peptide aa 24-37 (data not shown). These data imply that retinoschisin aggregates as multimers or complexes with other proteins of the membrane and that it forms a nonreducible aggregate in the cytosol. However, the possibility that the antibody recognizes another protein migrating at $65 \mathrm{kDa}$ cannot be ruled out.

Western blot analyses showed that the $27 \mathrm{kDa}$ retinoschisin was present in the cultured retinas and eyecups, whereas the $65 \mathrm{kDa}$ protein was detected at a low level not only in those tissues but also in cultured anterior segments, including mainly corneas, irises, and ciliary bodies (Fig. 4A, right panel). At least two other proteins with molecular masses $>65 \mathrm{kDa}$ were observed in the lanes containing homoge-

sion of cell-specific genes. Xlrs 1 mRNA was detected in several individual cells expressing either $R T \alpha$ (nine rod cells) or $C T \alpha$ (six cone cells) but not in cells expressing the Müller cell marker CAII (eight cells); the RT-PCR results of three cells from each cell type are shown in Figure 3. Multiple retinal cells were collected in a single micropipette as the positive control (Fig. 3, PC). After nates of the cultured anterior segments and in lanes of the R/C/S homogenates (Fig. 4A, right panel). These data indicate either that retinoschisin forms complexes in these tissues or that the antibody recognizes in them other proteins with similar molecular masses. Retinoschisin that is reducible to the monomeric form seems to be present only in the retina. 


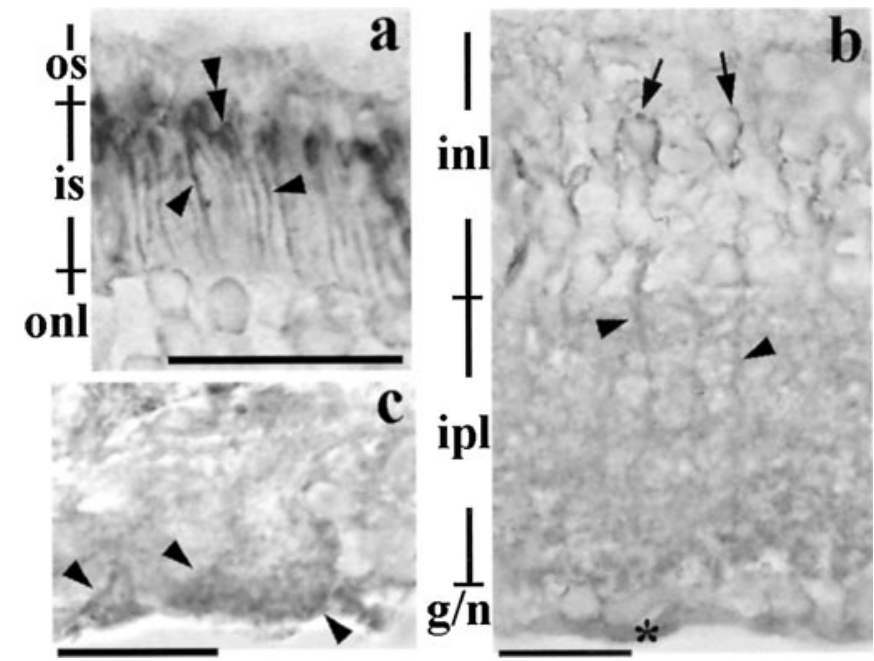

Figure 2. Retinoschisin in the adult retina. $a$, Retinoschisin-immunolabeling surrounds the inner segment of the photoreceptors (arrowheads) and is concentrated in the area adjacent to where the inner segment joins the outer segment (double arrowheads). $b$, Immunohistochemical reaction products in the inner retina appear around cell bodies (arrows) in the INL, processes in the INL and IPL (arrowheads), and in the nerve fiber layer (*). c, The immunoreaction products are also associated with the end feet of Müller cells (arrowheads) in the nerve fiber layer. Scale bar: $a, b, 20 \mu \mathrm{m} ; c, 13 \mu \mathrm{m}$. See Figure 1 for abbreviations.

\section{Secretion of retinoschisin}

Both the 27 and $65 \mathrm{kDa}$ proteins labeled by RS24-37 were found in the culture media of the eyecup and the retina but not of the anterior segment and the R/C/S (Fig. 4B). A photoreceptor secreted protein, IRBP, was also detected in these media; however, rod rhodopsin, a photoreceptor membrane protein, was not (Fig. $4 B$ ). These results indicate that the presence of retinoschisin in the media is not the consequence of its association with membrane debris loosened during incubation. From the data in Figure 4, $A$ and $B$, we can conclude that cells from cultured retinas and eyecups secrete retinoschisin.

As shown in Figure 4C, both the 27 and $65 \mathrm{kDa}$ proteins were present in the inner and outer retina of normal mice. They were also found in the 73- and 139-d-old $r d / r d$ retinas, consistent with the immunohistochemical data (Fig. 1). The monomeric retinoschisin was present in the culture medium of outer retinas. In contrast, no retinoschisin was detected in the culture media of inner retinas or $r d / r d$ retinas (Fig. $4 C$ ). Because the outer retinas have mainly photoreceptor cells, these data indicate that retinoschisin is secreted by photoreceptor cells and not by the inner retinal cells.

\section{Retinoschisin in dissociated retinal cells}

The findings described above-(1) localization of retinoschisin mRNA in photoreceptors (suggesting synthesis of the corresponding protein in these cells), (2) undetectable retinoschisin mRNA but presence of the protein in the inner retina, and (3) retinoschisin secretion presumably from photoreceptor cellsindicate that retinoschisin may be taken up by bipolar and Müller cells, or both, and transported by them to the vitreal border of the inner retina. Moreover, the secreted retinoschisin could also participate through its FA58C motif in adhesion/cell-cell interactions between the different retinal cells. For these reasons we examined the presence of retinoschisin inside and outside photoreceptor, bipolar, and Müller cells by immunostaining dissociated cells that had or had not been permeabilized with methanol. This treatment has been shown to remove some proteins local-

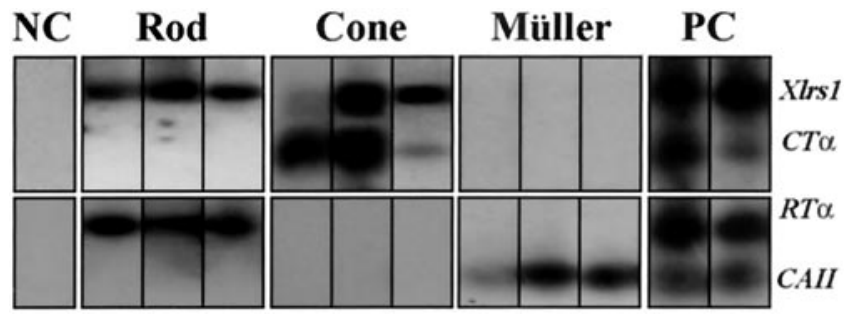

Figure 3. RT-PCR detection of XIrs $1 \mathrm{mRNA}$ expression in rod, cone, and Müller cells. Retinoschisin mRNA was found in individual cells expressing either the rod-specific $\alpha_{1}$-subunit of transducin $(R T \alpha)$ or the cone-specific $\alpha_{2}$-subunit of transducin $(C T \alpha)$, but not in cells expressing carbonic anhydrase II (CAII) mRNAs. The expression of these cell-specific genes was examined by RT-PCR using gene-specific primers and was used to reveal the identity of the isolated cells (rod, cone, and Müller cells, respectively). Expression of the above genes was not found in cells that were determined by morphology to be neither photoreceptors nor Müller cells [negative control (NC)]. Clusters containing multiple retinal cells were collected as the positive control $(P C)$, and expression of all four genes was detected in these samples.

ized to the cell surface [i.e., the lectin peanut agglutinin (Verdier et al., 2000)] and to allow the detection of intracellular proteins (Reid and Daw, 1995; Schipper et al., 1999). Consistent with these observations, we found that immunofluorescent staining of the cell-surface protein ROB was substantially reduced by methanol permeabilization (data not shown) and that intracellular CRALBP could be detected after methanol treatment. Therefore, this is a useful procedure to determine the localization of retinoschisin inside or outside different retinal cells.

Dissociated photoreceptor cells could be recognized by their small cell bodies ( $\sim 5 \mu \mathrm{m}$ diameter), each connected by a thin filament (the myoid) to the inner segment and the long, cylindrical outer segment (Fig. 5j,l). Without permeabilization, retinoschisin immunoreactivity was associated with the cell bodies and covered nearly the entire inner and outer segments of photoreceptor cells (Fig. $5 k, l$ ). After methanol permeabilization, the immunoreactivity became associated mainly with the inner/outer segment junction and much less with the cell body (Fig. 5i,j). This is similar to the pattern found in retinal sections (Fig. 2a) and consistent with the in situ results showing abundant retinoschisin mRNA in the inner segments (Fig. 1, P82). The levels of retinoschisin immunofluorescence associated with photoreceptor cells with or without methanol permeabilization were compared after normalization to "control" levels (obtained by using antibody preabsorbed with the peptide against which it was generated). Only levels significantly above (indicated by asterisks) the control level (0) were considered immunopositive (Fig. 6). ANOVA of immunofluorescence levels showed that retinoschisin was associated with photoreceptor cells $\left(F_{(1,248)}=63.505 ; p<0.001\right)$, regardless of which structure of the cell was examined $\left(F_{(1,248)}=\right.$ $0.160 ; p=0.690)$, but that this association was affected by the permeabilization procedure used (permeabilization factor: $F_{(1,248)}=7.558, p<0.01$; interaction between permeabilization and antibody treatment: $\left.F_{(1,248)}=7.561, p<0.01\right)$. A significantly higher than the control level of retinoschisin was found associated with nonpermeabilized cells at the soma $(p<0.001)$ and inner-outer segment region $(p<0.001)$, indicating localization of retinoschisin outside the cell bodies and the innerouter segments (Figs. 5k, 6A). Methanol permeabilization significantly reduced (by $60 \% ; p<0.05$ ) the level of retinoschisin at the soma, and this level was no longer different from that of the control $(p=0.166)$ (Fig. 6A). Although methanol changed the staining pattern of the inner-outer segment, it did not significantly affect the level of retinoschisin at this region $(p=0.347)$, 


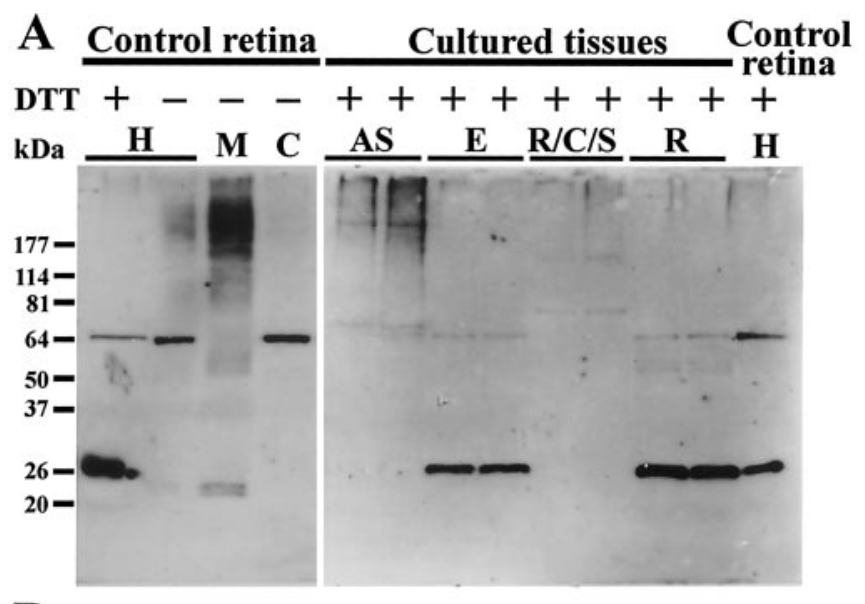

B Culture media
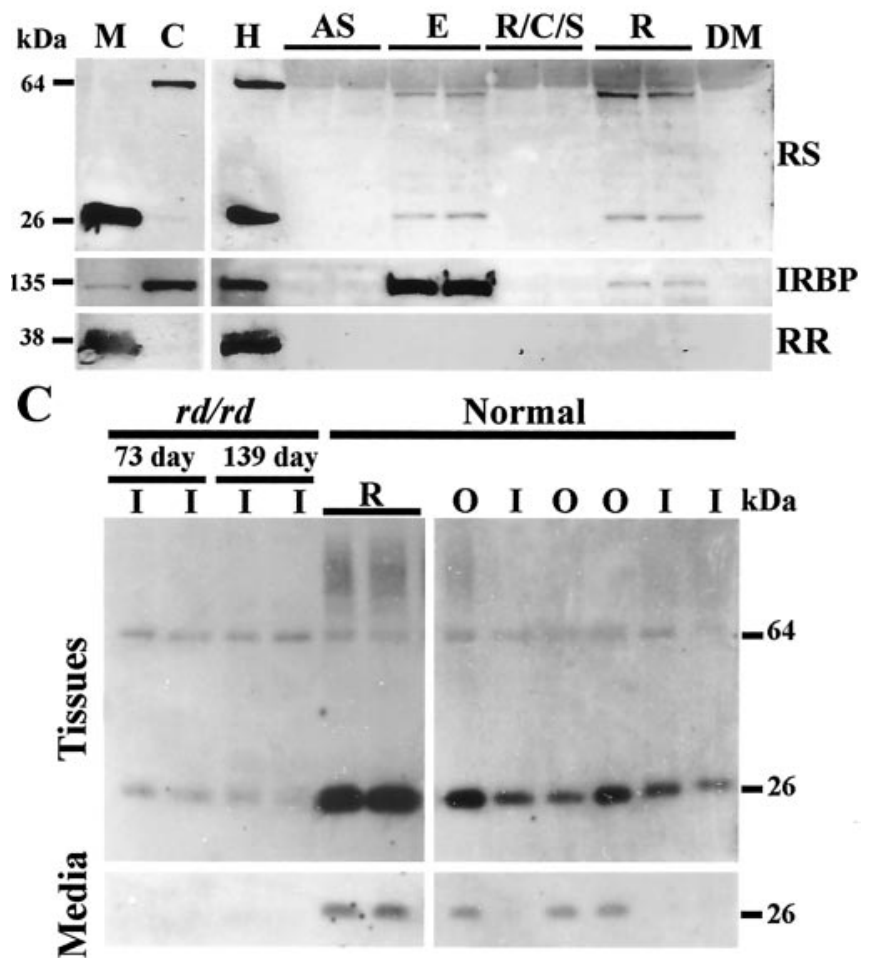

Figure 4. Retinoschisin in normal mouse retinal extracts and its secretion from different ocular tissues analyzed by Western blots. Proteins from all samples were subjected to SDS-PAGE. Western blots were labeled with the RS24-37 antibody. A, Left panel, Retinoschisin from a mouse retinal homogenate $(H)$, with or without the addition of DTT, and nonreduced membrane (M) and cytosol (C) fractions (see Materials and Methods). Right panel, Proteins from cultured eye tissues incubated with DTT. AS, Anterior segment; $E$, eyecup; R/C/S, retinal pigment epithelium/choroid/sclera; $R$, retina. $B$, Left panel, Retinal cellular fractions treated with DTT. Right panel, Retinal homogenate used as reference and the culture media of ocular tissues in $A$ after treatment with DTT. C, Left panel, Cultured adult $r d / r d$ retinas, containing only the inner layers, and normal retina (top panel); culture media of above samples (bottom panel). Right panel, Microdissected inner and outer layers of normal retinas (top panel) and their culture media (bottom panel). The $27 \mathrm{kDa}$ protein was observed only under reducing conditions in retinal samples and their culture media (see Results for details). IRBP, a predominately cytosolic protein, was used as a positive control for secretion and rod rhodopsin (RR), an intrinsic membrane protein, was used as a negative control. RS24-37 antibody also labeled a $65 \mathrm{kDa}$ protein present in the retinal cytosol. DM, DMEM culture medium; RS, retinoschisin; I, inner retina; 0 , outer retina.

which remained significantly above the control $(p<0.01)$. Taken together, these observations demonstrate that methanol removes extracellular retinoschisin associated with photorecep- tor cells mainly around the soma and the entire inner-outer segment but that retinoschisin is also located inside the inner segment.

ROB-positive bipolar cells all had a thin, smooth axon and tufted, crown-shaped dendrites (Figs. $5 a, b$ ) and did not immunoreact with an antibody against CRALBP, a Müller cell marker (Fig. 5c,d). CRALBP-positive Müller cells had two relatively thick, craggy processes extending out in opposite directions; the end of the proximal process formed the enlarged end foot (Fig. $5 e, f, r, s, w, x)$. Müller cells were not labeled by the ROB antibody (Fig. $5 g, h, q, v)$. Because the $\mathrm{ROB}$ antibody recognizes a cell surface antigen (Onoda and Fujita, 1987), both nonpermeabilized (Fig. $5 n$ ) and proteinase K-permeabilized bipolar cells (Fig. 5b) were immunolabeled by this antibody. In contrast, the CRALBP antibody labeled either Triton X-100 (Fig. 5f,w) or methanolpermeabilized Müller cells (Fig. $5 r$ ) but did not immunostain nonpermeabilized Müller cells (Fig. $5 u$ ). This pattern of staining agrees with CRALBP being an intracellular protein (Eisenfeld et al., 1985).

To examine the association of retinoschisin with bipolar and Müller cells, we performed sequential immunostaining of these cells with antibodies against retinoschisin, ROB, and CRALBP. The anti-retinoschisin antibody labeled nonpermeabilized cells that exhibited morphological characteristics of bipolar cells (Fig. $5 m, o$ ), and the same cells were also labeled by the ROB antibody (Fig. 5n). Quantitative analyses were performed on bipolar cells immunostained only for retinoschisin. These analyses showed that retinoschisin was associated with them (ANOVA; $F_{(1,393)}=$ 47.068; $p<0.001)$ regardless of which region of the cell was examined $\left(F_{(1,393)}=0.670 ; p=0.512\right)$, but this association was dependent on the permeabilization procedure (permeabilization effect: ANOVA; $F_{(1,393)}=25.728, p<0.001$; interaction with antibody treatment: ANOVA; $\left.F_{(1,393)}=25.726, p<0.001\right)$. Post hoc analyses revealed that retinoschisin was associated with the soma ( $p<0.001)$, axon $(p<0.05)$, and dendrites $(p<0.001)$ of only nonpermeabilized bipolar cells (Fig. $6 B$ ). Permeabilization significantly reduced retinoschisin immunofluorescence by $86-$ $97 \%$ at the soma and dendrites ( $p<0.001$ for both), and the levels of retinoschisin were no longer above the control levels at all three regions of the bipolar cell (Fig. $6 \mathrm{~B}$ ). These results show that retinoschisin is located mainly outside the bipolar cells and is removable by methanol. We corroborated this localization and the effect of methanol by triple labeling dissociated bipolar cells and confocal microscopy analysis. The retinoschisin immunostaining pattern of nonpermeabilized bipolar cells was nearly identical to that obtained by labeling cell plasma membranes with FM 1-43 but was different from cytosol labeling with CMAC (Fig. 7). In agreement with our quantitative analyses, methanol permeabilization abolished the retinoschisin immunoreactivity of bipolar cells (Fig. 7).

Sequential immunostaining demonstrated that cells with characteristics of Müller cells (Fig. 5e,f) were immunopositive for retinoschisin with or without methanol permeabilization (Fig. $5 p, t)$. The same cells were also immunopositive for CRALBP (Fig. $5 r, w$ ) but not for ROB antibodies (Fig. $5 q, v$ ). ANOVA of immunofluorescence levels showed a significant effect of antibody $\left(F_{(1,652)}=236.433 ; p<0.001\right)$ but no permeabilization effect $\left(F_{(1,652)}=1.533 ; p=0.216\right)$ and no difference between regions of the cell $\left(F_{(1,652)}=0.451 ; p=0.717\right)$, indicating that retinoschisin is associated with all structures of Müller cells regardless of permeabilization. Post hoc analyses revealed that permeabilization had no effect on the level of retinoschisin at the soma, the proximal process, and the end foot, but resulted in an increase in the 


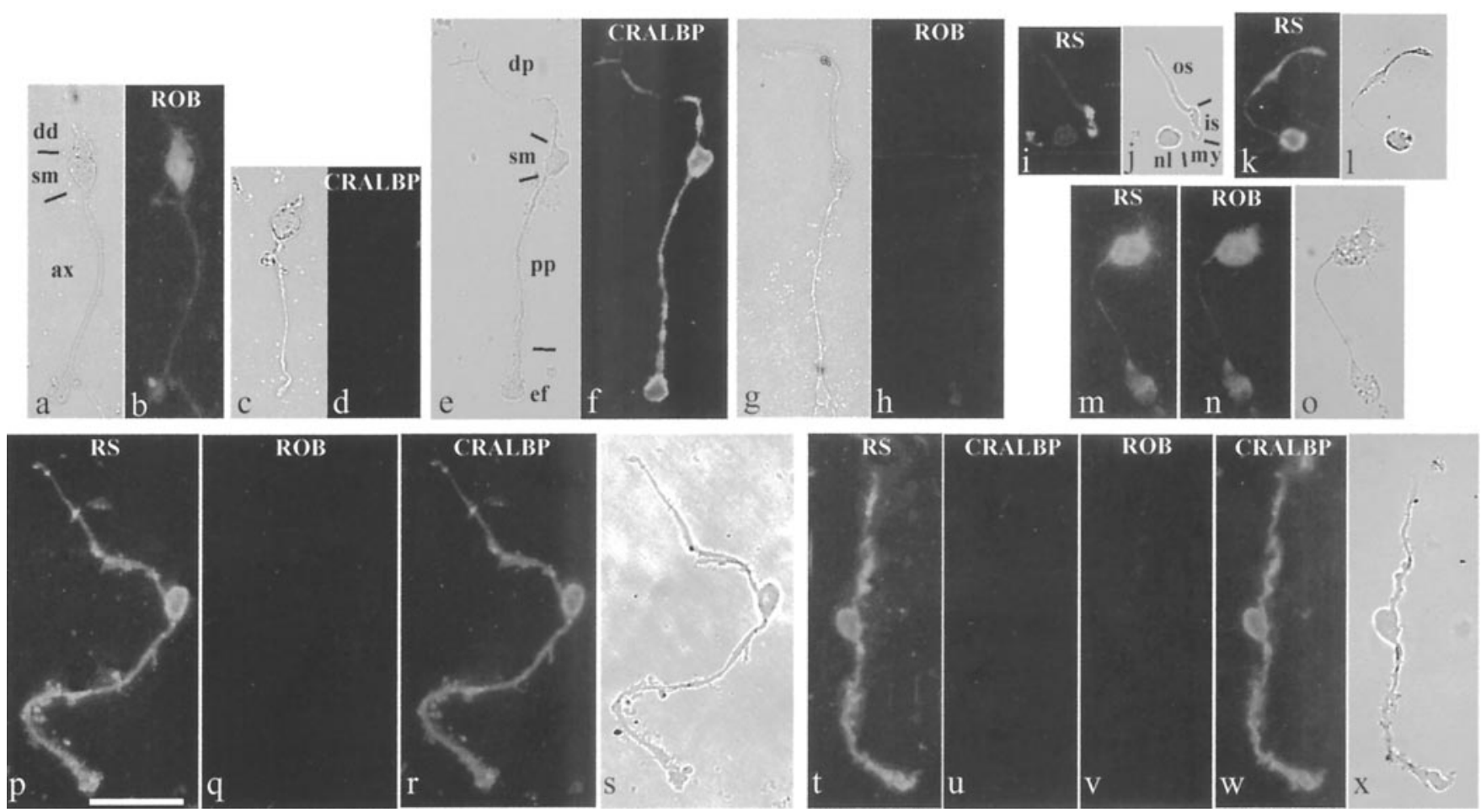

Figure 5. Retinoschisin in photoreceptor cells and marker-identified bipolar and Müller cells. The bipolar cell shown ( $a$ ) was recognized by the ROB antibody when permeabilized with proteinase $K(b)$; bipolar cells were not immunopositive for the Müller cell protein CRALBP $(c, d)$. Müller cells were identified by an antibody against CRALBP after Triton X-100 permeabilization $(e, f)$ and were not labeled by ROB $(g, h)$. Cells in $a$ and $b$ and $g$ and $h$ were immunostained at the same time and so were cells in c and $d$ and $e$ and $f$. The retinoschisin antibody (RS) labeled photoreceptor cells that were either permeabilized with methanol $(i, j)$ or not permeabilized $(k, I)$. Bipolar cells $(m-0)$ and Müller cells ( $p-s$ and $t-x)$ that had been immunostained for retinoschisin were subjected to sequential immunostainings, and the image acquisition after each staining was completed before the next round of staining. The nonpermeabilized bipolar cell in $m$ was also labeled by the ROB antibody $(n)$. The methanol-permeabilized Müller cell in $p$ was not labeled by ROB $(q)$ but was labeled by the antibody against CRALBP $(r)$. The nonpermeabilized Müller cell in $t$ was not labeled by the antibody against the intracellular protein CRALBP $(u)$. After Triton X-100 permeabilization, this Müller cell was not positive for ROB ( $v)$, but it was labeled by the CRALBP antibody ( $w$ ). $a, c, e, g$, $j, l, 0, s$, and $x$ were taken with bright field under high contrast. $b$ and $h, d$ and $f, q$ and $r$, and $u-w$ were acquired with the same exposure time. dd, Dendrite; sm, soma; ax, axon; dp, distal process; pp, proximal process; ef, end foot; os, outer segment; is, inner segment; my, myoid; nl, nucleus. Scale bar, $25 \mu \mathrm{m}$.

retinoschisin level at the distal process $(p<0.05)$ (Fig. $6 C$ ). Triple labeling and confocal microscopy analyses were also used to corroborate the localization of retinoschisin and the effect of methanol permeabilization on Müller cells. Retinoschisin immunolabeling of either the nonpermeabilized or the methanolpermeabilized cells was nearly identical to the FM 1-43 staining of cell membranes but different from the CMAC staining of the cytosol (Fig. 7). Staining of nonpermeabilized cells showed extracellular localization of retinoschisin. Similar levels of retinoschisin before and after methanol permeabilization suggest that retinoschisin may be resistant to removal from the cell surface, as it occurs with membrane-associated proteins, e.g., glutamate receptors (Reid et al., 1995). Alternatively, methanol removal of retinoschisin from outside Müller cells may allow the detection of retinoschisin associated with the inner domain of the cell membrane. Either of these two possibilities leads to the conclusion that retinoschisin is associated with the cell membrane of Müller cells. In addition, the increased retinoschisin level in the distal processes after permeabilization indicates an intracellular localization of retinoschisin in Müller cells.

\section{Discussion}

Retinoschisin and its mRNA are distributed differently in the mouse retina throughout postnatal life: the mRNA required for protein synthesis resides in the outer retina, where the photoreceptor cells and distal processes of Müller cells are located, whereas retinoschisin is in all retinal layers. More precisely, we demonstrated in single-cell RT-PCR experiments that retinoschisin mRNA is located only in cone and rod cells but not in Müller cells. Thus, de novo synthesis of retinoschisin does not occur in Müller cell processes. The localization of retinoschisin in photoreceptors is consistent with the results of studies showing that the gene encoding this protein is regulated by $\mathrm{Crx}$ (Livesey et al., 2000), a transcription factor that is crucial for photoreceptor differentiation (Chen et al., 1997; Furukawa et al., 1997). The lack of detectable retinoschisin mRNA in the inner retina supports the notion of retinoschisin being secreted into these retinal layers, an idea already inferred by the presence of a secretory leader peptide in its amino acid sequence (Sauer et al., 1997; Reid et al., 1999) and confirmed by our detection of retinoschisin in the medium of cultured photoreceptor-containing outer retina (Fig. 4).

To corroborate the site of retinoschisin synthesis, we measured the levels of its mRNA in retinas of $r d / r d$ mice at different times during postnatal development. We found that at 4 weeks of age, the rodless $r d / r d$ retina has less mRNA than that of the agematched normal retina. As cones degenerate, there is a progressive decrease in retinoschisin mRNA that becomes undetectable by $8-10$ postnatal weeks on Northern blots (Reid et al., 1999) (confirmed by Molday et al., 2001) or by in situ hybridization (Fig. 1). In contrast, retinoschisin is present in retinas of 8- to 10-week-old $r d / r d$ mice (Fig. $1 B$ ) (Molday et al., 2001), although its level is lower than normal, and it declines further in the next 10 weeks as cones continue to degenerate (Fig. $1 B$ ). These results 


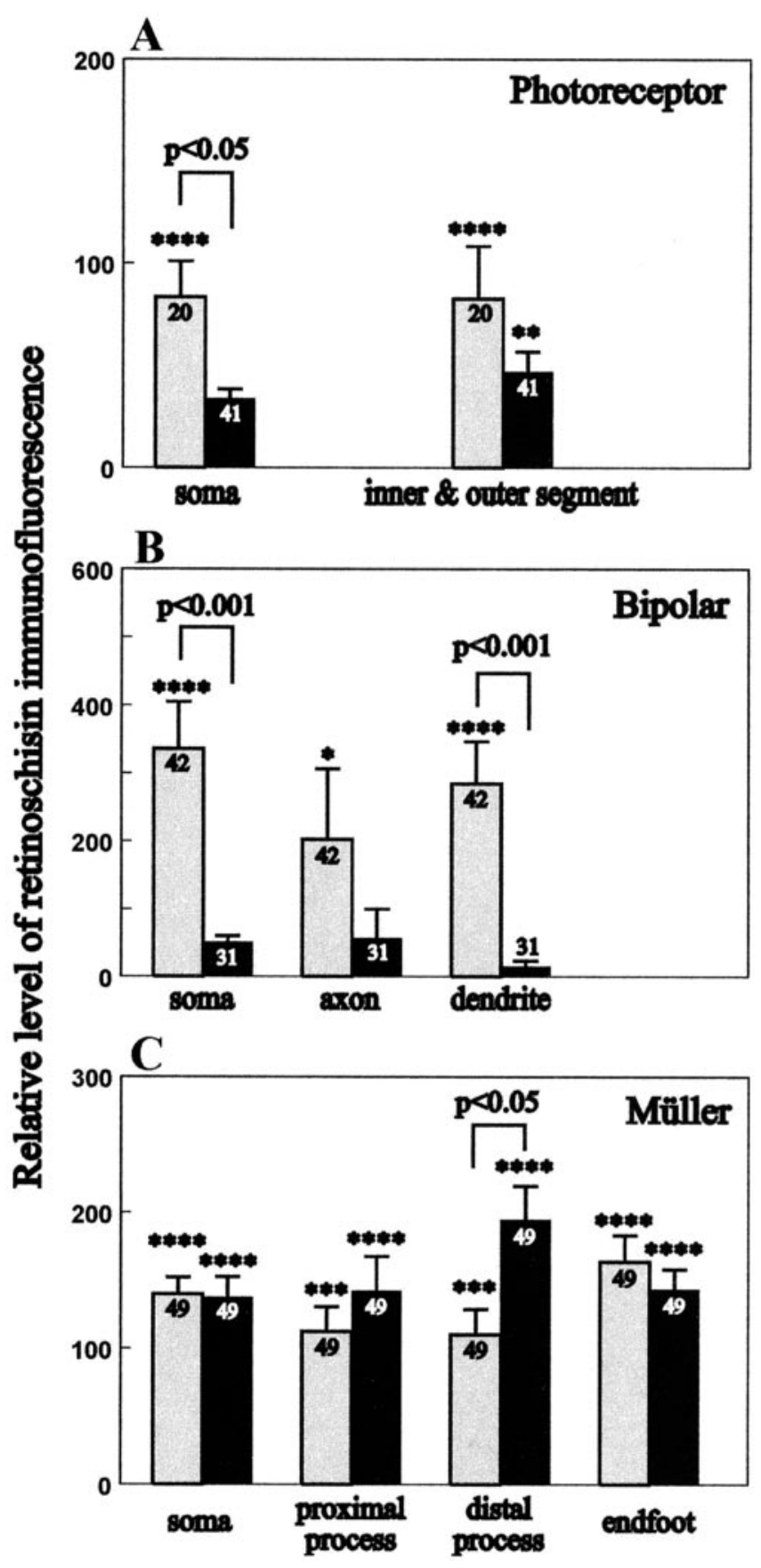

Figure 6. Relative levels of retinoschisin immunofluorescence in nonpermeabilized and methanol-permeabilized photoreceptor $(A)$, bipolar $(B)$, bipolar, and Müller $(C)$ cells. Immunofluorescent images of retinal cells were analyzed from confocal optical sections recorded under identical conditions. The relative level of retinoschisin immunofluorescence represents the percentage difference from the control. The immunofluorescence of the control was obtained using the anti-retinoschisin antibody preabsorbed with the peptide used to generate it. A lack of difference between the antibody staining of the sample and the control was given a value of 0 for the relative retinoschisin immunofluorescence. Bars represent the averaged relative retinoschisin immunofluorescence \pm the corresponding SEM for the number of observations performed (indicated in the bar). Gray bars represent nonpermeabilized cells; black bars represent methanol-permeabilized cells. Statistical comparisons between cells treated with retinoschisin antibody and the peptide preabsorbed antibody are indicated with asterisks only for those that are at a significant plevel: ${ }^{*} p<0.5$; $^{* *} p<0.01$; $^{* * *} p<0.005 ;{ }^{* * * *} p<0.0001$. The relative level of retinoschisin immunofluorescence is higher without than with permeabilization for the soma of photoreceptor cells $(p<0.05)$ and the soma $(p<0.001)$ and dendrite $(p<0.001)$ of bipolar cells. The level was significantly higher after permeabilization at the distal process of Müller cells $(p<0.05)$. substantiate the fact that retinoschisin is made in the photoreceptor cells. The cone cells that remain in 8-week-old $r d / r d$ retinas (LaVail et al., 1997) may have been the source of retinoschisin mRNA that was amplified by RT-PCR and gave a weak signal in the study of Molday et al. (2001).

Immunostaining of retinal sections showed clearly that retinoschisin is in the inner and outer retina (Figs. 1, 2) (Grayson et al., 2000; Molday et al., 2001; Weber et al., 2002), predominantly associated with photoreceptor inner segments, also present in the ONL, the INL, and the plexiform layers, and patchy in the NFL (Figs. 1, 2) (Grayson et al., 2000). Recently, Molday et al. (2001) reported that retinoschisin is present in bipolar but not Müller cells. We have confirmed the association of retinoschisin with the surface of bipolar cells (Figs. 6, 7). However, we also have found retinoschisin-immunopositive Müller cell end feet along the inner limiting membrane (Fig. 2b,c) and have observed immunoreactivity for retinoschisin in dissociated Müller cells (Figs. 5-7). In fact, the patchy staining of retinoschisin along the inner limiting membrane is also apparent in Figures $3 B, 4 A$, and $6 E$ of Molday et al. (2001) and in Figure $6 b$ of Weber et al. (2002). Permeabilization with methanol (in our study) instead of Triton X-100 (Molday et al., 2001) may have facilitated our detection of Müller cell retinoschisin. There is no obvious reason to suspect that the antibodies used by the two laboratories recognized different proteins. Both antibodies were generated against peptides of nearly identical sequences (Grayson et al., 2000; Molday et al., 2001), were affinity purified, and bound a membrane-associated protein of similar molecular mass. Although our antibody also recognized a cytosolic protein of $65 \mathrm{kDa}$ [not reported by Molday et al. (2001) ], the labeling of Müller cells is associated mainly with their plasma membranes, which do not have the $65 \mathrm{kDa}$ retinoschisin (Figs. 4, 7).

The amino acid sequence of retinoschisin contains the FA58C/discoidin motif that has been implicated in cell-cell interaction and adhesion (Sauer et al., 1997; Reid et al., 1999). The detection of retinoschisin on the outside surface of bipolar and Müller cells lends support to its involvement in these functions. Through cell-cell interactions or adhesion between retinal cells, or both, retinoschisin may contribute to maintain the integrity of the cytoarchitecture of the retina.

Both retinoschisin mRNA and protein appear at P5, a time when the inner segment of the photoreceptors has started to develop (Olney, 1968; Blanks et al., 1974) and that coincides with the expression of some phototransduction proteins (Bowes et al., 1988; Szél et al., 1994). The OPL seems to impose a boundary for retinoschisin mRNA but not for the protein. Retinoschisin can be seen spreading inwardly into the IPL and the NFL at P7, when immature blood vessels (Caley et al., 1972) and Müller cell end feet (Edwards et al., 1990) first appear. At the same time, rod bipolar cells and $\mathrm{ON}$-cone bipolar cells are differentiating, as indicated by the expression of metabotropic glutamate receptor subtype 6 (a major glutamate receptor of these cells) (Ueda et al., 1997). Thus, retinoschisin appears when retinal cells differentiate and when two nuclear layers segregate.

Müller, rod, bipolar, and some amacrine cells arise during a late proliferative phase (Young, 1985) and together, guided by Müller cells, form radial columns in the retina (Meller and Tetzkaff, 1976; Layer et al., 1997; Reese et al., 1999). Starting at P4, many cone nuclei are pushed deep into the ONL, and earlygenerated rod cells are pushed into the future INL. They need to migrate back scleradly (Young, 1984; Rich et al., 1997). The idea 
that Müller cells aid in this migration has been supported by the finding of displaced photoreceptor cells in vivo and in culture when Müller cells have been damaged (Rich et al., 1995; Willbold et al., 1995). Interestingly, some XLRS retinas have either fewer or no photoreceptor nuclei in the macula (Manschot, 1972; Ando et al., 2000), lack a well defined columnar organization in the ONL, or have displaced cone cells in the inner retina (Yanoff et al., 1968; Condon et al., 1986). Similarly, Xlrs1 inactivation in mice reduces photoreceptor cell number and causes photoreceptor cell displacement (Weber et al., 2002), as in the case of Müller cell disruption (Rich et al., 1995). Furthermore, both nuclear layers become poorly segregated, and inner retinal cells are disorganized (Weber et al., 2002). The abnormalities in humans could result from inappropriate interactions between photoreceptors/neurons and Müller cells mediated by the defective protein produced by the mutated XLRS1 gene, and in the knock-out mice from the lack of retinoschisin. A defective CRALBP immunolabeling of Müller cell end feet is also detected in the knock-out retina, without schisis in the NFL (Weber et al., 2002). The formation of the schisis may be a complicated process, involving several factors in addition to the participation of retinoschisin in the adhesion of the NFL to the inner retinal layers. More comprehensive studies of the effect of knocking out Xlrs 1 are required to establish or rule out Müller cell involvement in XLRS.

Physiological evidence has suggested that the bipolar pathway is defective in XLRS (Alexander et al., 2000; Shinoda et al., 2001). Association of retinoschisin with bipolar dendrites is consistent with its involvement in information processing along the bipolar pathway and in the development of this pathway. The Xlrs 1 knock-out mice have displaced synaptic layers and severely attenuated synaptic formation in the IPL (Weber et al., 2002). Because retinoschisin appears before the formation of bipolar cell synapses in the OPL and the IPL (Olney, 1968; Blanks et al., 1974), it may guide the bipolar axons and dendrites to establish proper neural connections, as is the case with another FA58C/ discoidin-containing protein, neuropilin (He and Tessier-Lavigne, 1997; Kolodkin et al., 1997).

Photoreceptors are the last to mature in the chain of cells necessary to enable transmission of visual information to the brain, but they are the first to participate in the biochemical cascade conveying light information. Many events occur from the time of photoreceptor differentiation to the time of eye opening, and they need to be synchronized in the absence of visual inputs. The use of extracellular signaling molecules may be a way to achieve this coordination. The presence of retinoschisin during retinal development and its association with bipolar and Müller cells seems to place this protein in the right position at the right time.
In summary, our results suggest that the photoreceptorsecreted retinoschisin mediates a complicated set of interactions among photoreceptor, bipolar and Müller cells, and nerve fibers. Possibly, it may participate in the coordination of retinal development, and through its adhesion properties it may also be involved in the maintenance of the cytoarchitecture and normal electrophysiology of the retina.

\section{References}

Alexander KR, Fishman GA, Grover S (2000) Temporal frequency deficits in the electroretinogram of the cone system in X-linked retinoschisis. Vision Res 40:2861-2868.

Ando A, Takahashi K, Sho K, Matsushima M, Okamura A, Uyama M (2000) Histopathological findings of X-linked retinoschisis with neovascular glaucoma. Graefe's Arch Clin Exp Ophthalmol 238:1-7.

Apweiler R, Attwood TK, Bairoch A, Bateman A, Birney E, Biswas M, Bucher P, Cerutti L, Corpet F, Croning MDR, Durbin R, Falquet L, Fleischmann W, Gouzy J, Hermjakob H, Hulo N, Jonassen I, Kahn D, Kanapin A, Karavidopoulou Y, et al. (2001) The InterPro database, an integrated documentation resource for protein families, domains and functional sites. Nucleic Acids Res 29:37-40. 
Baumgartner S, Littleton JT, Broadie K, Bhat MA, Harbecke R, Lengyel JA, Chiquet-Ehrismann R, Prokop A, Bellen HJ (1996) A Drosophila neurexin is required for septate junction and blood-nerve barrier formation and function. Cell 87:1059-1068.

Blanks JC, Adinolfi AM, Lolley RN (1974) Synaptogenesis in the photoreceptor terminal of the mouse retina. J Comp Neurol 156:81-94.

Bowes C, van Veen T, Farber DB (1988) Opsin, G-protein and 48-kDa protein in normal and $r d$ mouse retinas: developmental expression of mRNAs and proteins and light/dark cycling of mRNAs. Exp Eye Res 47:369-390.

Caley DW, Johson C, Liebelt RA (1972) The postnatal development of the retina in the normal and rodless CBA mouse: a light and electron microscopic study. Am J Anat 133:179-212.

Chen S, Wang OL, Nie Z, Sun H, Lennon G, Copeland NG, Gilbert DJ, Jenkins NA, Zack DJ (1997) Crx, a novel Otx-like paired-homeodomain protein, binds to and transactivates photoreceptor cell-specific genes. Neuron 19:1017-1030.

Condon GP, Brownstein S, Wang N, Kearns AF, Ewing CC (1986) Congenital hereditary (juvenile X-linked) retinoschisis: histopathologic and ultrastructural findings in three eyes. Arch Ophthalmol 104:576-583.

Edwards MA, Yamamoto M, Caviness VSJR (1990) Organization of radial glia related cells in the developing murine CNS. An analysis based on a new monoclonal antibody marker. Neuroscience 36:121-144.

Einheber S, Zanazzi G, Ching W, Scherer S, Milner TA, Peles E, Salzer JL (1997) The axonal membrane protein Caspr, a homologue of neurexin IV, is a component of the septate-like paranodal junctions that assemble during myelination. J Cell Biol 139:1495-1506.

Eisenfeld AJ, Bunt-Milam AH, Saari JC (1985) Localization of retinoidbinding proteins in developing rat retina. Exp Eye Res 41:299-304.

Furukawa T, Morrow EM, Cepko CL (1997) Crx, a novel otx-like homebox gene, shows photoreceptor-specific expression and regulates photoreceptor differentiation. Cell 91:531-541.

George NDL, Yates JRW, Moore AT (1995) X-linked retinoschisis. Br J Ophthalmol 79:697-702.

Gobron S, Monnerie H, Meiniel R, Creveaux I, Lehmann W, Lamalle D, Dastugue B, Meiniel A (1996) SCO-spondin: a new member of the thrombosposin family secreted by the subcommissural organ is a candidate in the modulation of neuronal aggregation. J Cell Sci 109:1053-1061.

Grayson C, Reid SNM, Ellis JA, Rutherford A, Sowden JC, Yates JRW, Farber DB, Trump D (2000) Retinoschisin, the X-linked retinoschisis protein, is a secreted photoreceptor protein, and is expressed and secreted by differentiated Weri cells. Human Mol Genet 9:1873-1879.

Haverkamp S, Wässle H (2000) Immunocytochemical analysis of the mouse retina. J Comp Neurol 424:1-23.

He Z, Tessier-Lavigne M (1997) Neuropilin is a receptor for the axonal chemorepellent semaphorin III. Cell 90:739-751.

Ishii M, Horio Y, Tada Y, Hibino $\mathrm{H}$, Inanobe A, Ito M, Yamada M, Botow T, Uchiyama Y, Kurachi Y (1997) Expression and clustered distribution of an inwardly rectifying potassium channel, $\mathrm{K}_{\mathrm{AB}}-2 / \mathrm{Kir} 4.1$, on mammalian retinal Müller cell membrane: their regulation by insulin and laminin signals. J Neurosci 17:7725-7735.

Kitsukawa T, Shimizu M, Sanbo M, Hirata T, Taniguchi M, Bekku Y, Yagi T, Fujisawa H (1997) Neuropilin-semaphorin III/D-mediated chemorepulsive signals play a crucial role in peripheral nerve projection in mice. Neuron 19:995-1005.

Kolodkin AL, Levengood DV, Rowe EG, Tai YT, Giger RJ, Ginty DD (1997) Neuropilin is a semaphorin III receptor. Cell 90:753-762.

Kumazaki T, Hamada K, Mitsui Y (1994) Detection of mRNA expression in a single cell by direct RT-PCR. Biotechniques 16:1017-1019.

LaVail MM, Matthes MT, Yasumura D, Steinberg RH (1997) Variability in rate of cone degeneration in the retinal degeneration $(r d / r d)$ mouse. Exp Eye Res 65:45-50.

Layer PG, Berger J, Kinkl N (1997) Cholinesterases precede "ON-OFF" channel dichotomy in the embryonic chick retina before onset of synaptogenesis. Cell Tissue Res 288:407-416.

Livesey FJ, Furukawa T, Steffen MA, Church GM, Cepko CL (2000) Microarray analysis of the transcriptional network controlled by the photoreceptor homeobox gene Crx. Curr Biol 10:301-310.
Manschot W (1972) Pathology of hereditary juvenile retinoschisis. Arch Ophthalmol 88:131-138.

Marín O, Yaron A, Bagri A, Tessier-Lavigne M, Rubenstein JLR (2001) Sorting of striatal and cortical interneurons regulated by semaphorinneuropilin interactions. Science 293:872-875.

Meller K, Tetzkaff W (1976) Scanning electron microscopic studies on the development of the chick retina. Cell Tissue Res 170:145-159.

Molday LL, Hicks D, Sauer CG, Weber BHF, Molday RS (2001) Expression of X-linked retinoschisis protein RS1 in photoreceptor and bipolar cells. Invest Ophthalmol Vis Sci 42:816-825.

Muñoz-Barroso I, Durell S, Sakaguchi K, Appella E, Blumenthal R (1998) Dilation of the human immunodeficiency virus-1 envelope glycoprotein fusion pore revealed by the inhibitory action of a synthetic peptide from gp41. J Cell Biol 140:315-323.

Olney JW (1968) An electron microscopic study of synapse formation, receptor outer segment development, and other aspects of developing mouse retina. Invest Ophthalmol 7:250-268.

Onoda N, Fujita SC (1987) A monoclonal antibody specific for a subpopulation of retinal bipolar cells in the frog and other vertebrates. Brain Res 416:359-363.

Peachey NS, Fishman GA, Derlacki DJ, Brigell MG (1987) Psychophysical and electroretinographic findings in X-linked juvenile retinoschisis. Arch Ophthalmol 105:513-516.

Peles E, Nativ M, Lustig M, Grumet M, Schilling J, Martinez R, Plowman GD, Schlessinger J (1997) Identification of a novel contactin-associated transmembrane receptor with multiple domains implicated in proteinprotein interactions. EMBO J 16:978-988.

Peterson GL (1977) A simplification of the protein assay method of Lowry et al. which is more generally applicable. Anal Biochem 83:346-365.

Reese BE, Necessary BD, Tam PPL, Faulkner-Jones B, Tan S-S (1999) Clonal expansion and cell dispersion in the developing mouse retina. Eur J Neurosci 11:2965-2978.

Reid SNM, Daw NW (1995) Dark-rearing changes dendritic microtubuleassociated protein 2 (MAP2) but not subplate neurons in cat visual cortex. J Comp Neurol 359:38-47.

Reid SNM, Romano C, Hughes T, Daw NW (1995) Immunohistochemical study of two phosphoinositide-linked metabotropic glutamate receptors (mGluR1 $\alpha$ and mGluR5) in the cat visual cortex before, during and after the peak of the critical period for eye specific connections. J Comp Neurol 355:470-477.

Reid SNM, Romano C, Hughes T, Daw NW (1997) Developmental and sensory-dependent changes of phosphoinositide-linked metabotropic glutamate receptors. J Comp Neurol 389:577-583.

Reid SNM, Akhmedov NB, Piriev NI, Kozak CA, Danciger M, Farber DB (1999) The mouse X-linked juvenile retinoschisis cDNA: expression in photoreceptors. Gene 227:257-266.

Rich KA, Figueroa SL, Zhan Y, Blanks JC (1995) Effects of Muller cell disruption on mouse photoreceptor cell development. Exp Eye Res 61:235-248.

Rich KA, Zhan Y, Blanks JC (1997) Migration and synaptogenesis of cone photoreceptors in the developing mouse retina. J Comp Neurol 388:47-63.

Sampath AP, Matthews HR, Cornwall MC, Fain GL (1998) Bleached pigment produces a maintained decrease in outer segment $\mathrm{Ca}^{2+}$ in Salamander rods. J Gen Physiol 111:53-64.

Sánchez MP, Tapley P, Saini SS, He B, Pulido D, Barbacid M (1994) Multiple tyrosine proteins in rat hippocampal neurons: isolation of Ptk-3, a receptor expressed in proliferative zones of the developing brain. Proc Natl Acad Sci USA 91:1819-1823.

Sauer CG, Gehrig A, Warneke-Wittstock R, Marquardt A, Ewing CC, Gibson A, Lorenz B, Jurklies B, Weber BHF (1997) Positional cloning of the gene associated with $\mathrm{X}$-linked juvenile retinoschisis. Nat Genet 17:164-170.

Schipper RG, Romain N, Otten AA, Tan J, Lange WP, Verhofstad AAJ (1999) Immunocytochemical detection of ornithine decarboxylase. J Histochem Cytochem 47:1395-1404.

Shinoda K, Ohde H, Mashima Y, Inoue R, Ishida S, Inoue M, Kawashima S, Oguchi Y (2001) On- and off-responses of the photopic electroretinograms in X-linked juvenile retinoschisis. Am J Ophthalmol 131: $489-494$. 
Szél Á, van Veen T, Röhlich P (1994) Retinal cone differentiation. Nature 370:336.

The Retinoschisis Consortium (1998) Functional implications of the spectrum of mutations found in 234 cases with X-linked-juvenile retinoschisis. Human Mol Genet 7:1185-1192.

Ueda Y, Iwakabe H, Masu M, Suzuki M, Nakanishi S (1997) The mGluR6 5 ' upstream transgene sequence directs a cell-specific and developmentally regulated expression in retinal rod and ON-type cone bipolar cells. J Neurosci 17:3014-3023.

Verdier M, Jayat C, Ratinaud H, Troutaud D (2000) Optimization of cell permeabilization for multiparametric flow cytometric analysis with lectin staining. Cytometry 41:55-61.

Weber BHF, Schrewe H, Molday LL, Gehrig A, White KL, Seeliger MW, Jaissle GB, Friedburg C, Tamm E, Molday RS (2002) Inactivation of the murine $\mathrm{X}$-linked juvenile retinoschisis gene, Rs1h, suggests a role of retinos- chisin in retinal cell layer organization and synaptic structure. Proc Natl Acad Sci USA 99:6222-6227.

Whalley T, Terasaki M, Cho MS, Vogel SS (1995) Direct membrane retrieval into large vesicles after exocytosis in sea urchin eggs. J Cell Biol 131:1183-1192.

Willbold E, Reiniche M, Lance-Jones C, Lagenaur C, Lemmon V, Layer PG (1995) Müller glia stabilizes cell columns during retinal development: lateral cell migration but not neuropil growth is inhibited in mixed chickquail retinospheroids. Eur J Neurosci 7:2277-2284.

Yanoff M, Rahn EK, Zimmerman LE (1968) Histopathology of juvenile retinoschisis. Arch Ophthalmol 79:49-53.

Young RW (1984) Cell death during differentiation of the retina in the mouse. J Comp Neurol 229:362-373.

Young RW (1985) Cell differentiation in the retina of the mouse. Anat Rec 212:199-205 